\title{
Spa therapy in the treatment of knee osteoarthritis: a large randomised multicentre trial
}

\author{
R Forestier, ${ }^{1}$ H Desfour, ${ }^{2}$ J-M Tessier, ${ }^{3}$ A Françon, ${ }^{1}$ A M Foote, ${ }^{4}$ C Genty, ${ }_{1}^{4}$ C Rolland, ${ }^{4}$ \\ C-F Roques, ${ }^{5}$ J-L Bosson ${ }^{4}$
}

\begin{abstract}
- Additional data are published online only at http:// ard.bmj.com/content/volXX/ issueX
\end{abstract}

${ }^{1}$ Centre for Rheumatology and Balneotherapy Research, Aix Les Bains, France

2Private Practice, 13 Av des

Thermes Athéna, Balaruc les Bains, France

${ }^{3}$ Spa Hospital, Dax, France ${ }^{4}$ Clinical Research Centre, INSERM CIC03, Grenoble, France

5 University of Toulouse, Toulouse, France

\section{Correspondence to} Professor Jean-Luc Bosson, Clinical Research Centre, CHU de Grenoble BP 217, Grenoble 38043, France; jlbosson@ imag.fr

Accepted 23 July 2009 Published Online First

3 September 2009

\begin{abstract}
Objective To determine whether spa therapy, plus home exercises and usual medical treatment provides any benefit over exercises and usual treatment, in the management of knee osteoarthritis.

Methods Large multicentre randomised prospective clinical trial of patients with knee osteoarthritis according to the American College of Rheumatology criteria, attending French spa resorts as outpatients between June 2006 and April 2007. Zelen randomisation was used so patients were ignorant of the other group and spa personnel were not told which patients were participating. The main endpoint criteria were patient self-assessed. All patients continued usual treatments and performed daily standardised home exercises. The spa therapy group also received 18 days of spa therapy (massages, showers, mud and pool sessions).
\end{abstract}

Main Endpoint The number of patients achieving minimal clinically important improvement (MCII) at 6 months, defined as $\geq 19.9 \mathrm{~mm}$ on the visual analogue pain scale and/or $\geq 9$. 1 points in a normalised Western Ontario and McMaster Universities osteoarthritis index function score and no knee surgery.

Results The intention to treat analysis included 187 controls and 195 spa therapy patients. At 6 months, 99/195 (50.8\%) spa group patients had MCII and 68/187 (36.4\%) controls $\left(\chi^{2}=8.05 ; d f=1 ; p=0.005\right)$. However, no improvement in quality of life (Short Form 36) or patient acceptable symptom state was observed at 6 months.

Conclusion For patients with knee osteoarthritis a 3-week course of spa therapy together with home exercises and usual pharmacological treatments offers benefit after 6 months compared with exercises and usual treatment alone, and is well tolerated.

Trial registration number NCT00348777.

In Europe spa therapy is frequently prescribed for knee osteoarthritis. Of the 403381 patients receiving spa therapy for rheumatism in 2007 in France, nearly half presented with knee osteoarthritis. Spa therapy is reimbursed by the social security in France and in many other continental European countries.

Despite numerous small scale studies, high quality scientific evidence for the efficacy of spa therapy for knee osteoarthritis is lacking. In a recent Cochrane systematic review, even those studies that met the selection criteria were found to be flawed. ${ }^{1}$ Therefore, spa therapy does not figure in the recommended treatments of the European League Against Rheumatism ${ }^{2}$ or recent reviews. ${ }^{3}$

In this multicentre randomised controlled trial (RCT) we aimed to include enough patients to obtain sufficient statistical power to fill the gap in evidence-based data of high quality evaluating the use of spa therapy for knee osteoarthritis. Our primary objective was the therapeutic efficacy of spa therapy for knee osteoarthritis at 6 months in patients following usual treatments and a home exercise programme compared with a control group receiving usual treatments and a home exercise programme alone.

\section{PATIENTS AND METHODS}

This study was carried out in the three largest spa therapy resorts in France, Aix-les-Bains, Balaruc and Dax. In 2007, 29 000, 36000 and 50000 people, respectively, attended these three resorts for spa therapy. Patients were recruited locally, so that they could attend the centre on a daily basis, by advertisements in the regional press and posters in pharmacies and surgery waiting rooms. ${ }^{4}$ Recruitment notices referred to treatment for knee osteoarthritis but did not specify spa therapy.

Patients were enrolled by a trained examining physician in private practice outside and independent of the spa setting and with no vested interest in the spa. Osteoarthritis was confirmed by physical examination and the presence of osteophytes on the x-rays.

Inclusion criteria followed the definitions of the American College of Rheumatology: painful knee osteoarthritis plus either age greater than 50 years and/or morning stiffness lasting more than 30 minutes and/or articular crepitation. ${ }^{5}$ The evidence required was a knee x-ray examination in the past 3 years, including anteroposterior, schuss, lateral and skyline views to grade the severity of osteoarthritis, and pain intensity of $30 \mathrm{~mm}$ or greater on the visual analogue pain scale (VAS pain). ${ }^{6}$

Exclusion criteria were: osteoarthritis limited to the patellofemoral joint; severe depression or psychosis; a contraindication (immune deficiency, evolving cardiovascular conditions, cancer, infection) or intolerance to any aspect of spa treatment; professional involvement with a spa resort; spa treatment within the previous 6 months; knee intrajoint corticosteroid injection within the past 3 months; massages, physiotherapy or acupuncture in the past month; a non-steroidal antiinflammatory drug (NSAID) within the past 5 days or other analgesic drug in the previous $12 \mathrm{~h}$, or a change in symptomatic slow-acting drugs in osteoarthritis (SYSADOA) in the past 3 months.

\section{Intervention}

At inclusion the examining physician explained the home exercise programme to all patients and the online under the BMJ Journals locked scheme, see http 
importance of performing all four exercises, each six times, three times a day. ${ }^{7}$ All patients were given a booklet about knee osteoarthritis with details of the home exercise programme (see supplementary file 1, available online only) and continued their usual treatments (analgesics, NSAID, SYSADOA, physiotherapy). The self-assessment forms were completed without assistance in the waiting room.

In addition, the spa therapy group received 18 days of therapy over 3 weeks. The standardised knee osteoarthritis therapy programme was designed by experienced spa therapy physicians. Spa mineral water and treatments are approved and controlled by the French authorities. Treatment included: mineral hydrojet sessions at $37^{\circ} \mathrm{C}$ for 15 minutes, manual massages of the knee and thigh under mineral water at $38^{\circ} \mathrm{C}$ by a physiotherapist for 10 minutes, applications of mineral matured mud at $45^{\circ} \mathrm{C}$ to the knees for 15 minutes and supervised general mobilisation in a collective mineral water pool at $32^{\circ} \mathrm{C}$ in groups of six patients for 25 minutes.

Attendance, tolerance and proper performance of the various treatments were checked by an independent physician (general practitioner, rheumatologist or physiatrist) during consultations at the start, middle and end of the 3-week therapy period. Study patients were mixed with the general public and the centre personnel were not informed which patients were taking part in the clinical trial.

Patients in the control group were offered a 3-day wellness package at their local spa resort following the 6-month follow-up visit.

\section{Follow-up and data collection}

Follow-up was at 1, 3 and 6 months, by a visit to the examining physician who completed the electronic case report form, enquired whether the patient was doing the exercises and insisted on their importance. At each visit, but not in the presence of the physician, the patients filled in self-assessment of: their average level of pain over the previous 7 days on the VAS pain scale, ${ }^{6}$ the Western Ontario and McMaster Universities osteoarthritis index (WOMAC) ${ }^{9}$ and the quality of life questionnaire, Short Form 36 (SF36), ${ }^{10}$ without assistance. At 9 months these forms were completed at home and returned to the coordination centre by post.

The primary endpoint was achievement of a minimal clinically important improvement (MCII) ${ }^{11}$ defined as $19.9 \mathrm{~mm}$ or greater on the VAS pain ${ }^{6}$ scale and/or 9.1 points or greater on the WOMAC function subscale normalised to a 0-100 score, ${ }^{9}$ and no knee surgery, at 6 months. For WOMAC we used a five-point Likert scale for each item and higher scores indicated greater severity. ${ }^{11}$

Secondary endpoints were: patient acceptable symptom state, ${ }^{12}$ VAS pain $32 \mathrm{~mm}$ or less, normalised WOMAC function subscale 31 points or less; knee flexion, effusion and swelling; associated treatments; the overall opinions of the patient and the examining physician and quality of life.

All items collected by the examining physician at baseline and during follow-up visits are listed in table 1 and online tables (supplementary file 2, available online only), respectively. All adverse events were recorded.

\section{Design}

The sample size was determined using an open preliminary study with 13 consecutive patients, from which we calculated that $50 \%$ would be improved in the spa group and estimated that $25 \%$ would be improved in the control group. The agreed
Table 1 Baseline characteristics of participants

\begin{tabular}{|c|c|c|}
\hline Characteristics & Control & Spa therapy \\
\hline Male, n/N (\%) & $119 / 223(53.4)$ & $118 / 228(51.8)$ \\
\hline Age, mean $\pm S D(n)$ & $64.3 \pm 10.4(223)$ & $63.0 \pm 9.1(228)$ \\
\hline \multicolumn{3}{|l|}{ History of treatment for the knee } \\
\hline Medication, $\mathrm{n} / \mathrm{N}(\%)$ & $187 / 223(83.9)$ & $195 / 228(85.5)$ \\
\hline Massage, n/N (\%) & $70 / 223(31.4)$ & $73 / 228(32.0)$ \\
\hline Intra-articular injection, $\mathrm{n} / \mathrm{N}(\%)$ & $51 / 223(22.9)$ & $59 / 228(25.9)$ \\
\hline Hyaluronic acid treatment, $n / \mathrm{N}(\%)$ & $92 / 223(41.3)$ & $95 / 228(41.7)$ \\
\hline Surgery, n/N (\%) & $82 / 223(36.8)$ & $81 / 228(35.5)$ \\
\hline Previous spa therapy $\mathrm{n} / \mathrm{N}(\%)$ & $74 / 218(33.9)$ & $61 / 227(26.9)$ \\
\hline $\begin{array}{l}\text { Other physical treatment, } \mathrm{n} / \mathrm{N}(\%) \text { (brace, } \\
\text { traction, manipulation or physiotherapy in } \\
\text { past } 3 \text { months) }\end{array}$ & $10 / 223(4.5)$ & $10 / 228(4.4)$ \\
\hline \multicolumn{3}{|l|}{ Prognostic factors } \\
\hline $\begin{array}{l}\text { Length of present episode, in months, } \\
\text { mean } \pm S D(n)\end{array}$ & $63.9 \pm 73.3(223)$ & $60.5 \pm 72.0(228)$ \\
\hline Number of acute episodes, mean $\pm S D(n)$ & $7.8 \pm 10.5(223)$ & $8.6 \pm 16.9(228)$ \\
\hline Family history of osteoarthritis, $\mathrm{n} / \mathrm{N}(\%)$ & $116 / 223(52.0)$ & $117 / 228(51.3)$ \\
\hline Body mass index, mean $\pm S D(n)$ & $29.0 \pm 4.6(223)$ & $30.7 \pm 5.9(228)$ \\
\hline \multicolumn{3}{|l|}{ Knee examination } \\
\hline Knee joint swelling, $\mathrm{n} / \mathrm{N}(\%)$ & $94 / 223(42.2)$ & $96 / 228(42.1)$ \\
\hline Knee joint effusion, $\mathrm{n} / \mathrm{N}(\%)$ & $62 / 223(27.8)$ & $58 / 228(25.4)$ \\
\hline $\begin{array}{l}\text { Knee joint crepitation on active motion, } \\
\mathrm{n} / \mathrm{N}(\%)\end{array}$ & $107 / 223(48.0)$ & $107 / 228(46.9)$ \\
\hline \multicolumn{3}{|c|}{ Radiological severity (Kellgren and Lawrence), $\mathrm{n} / \mathrm{N}(\%)$} \\
\hline Grade 1 & $53 / 223(23.8)$ & $54 / 228(23.7)$ \\
\hline Grade 2 & $70 / 223(31.4)$ & $73 / 228(32.0)$ \\
\hline Grade 3 & $83 / 223(37.2)$ & $82 / 228(36.0)$ \\
\hline Grade 4 & $17 / 223(7.6)$ & $19 / 228(8.3)$ \\
\hline WOMAC pain score, 0-100, mean \pm SD (n) & $42.0 \pm 18.1(223)$ & $45.1 \pm 17.8(224)$ \\
\hline $\begin{array}{l}\text { WOMAC function score, } 0-100 \text {, } \\
\text { mean } \pm S D(n)\end{array}$ & $38.9 \pm 17.1(218)$ & $42.6 \pm 19.7(214)$ \\
\hline VAS pain, $0-100 \mathrm{~mm}$, mean $\pm S D(\mathrm{n})$ & $45.7 \pm 19.0(223)$ & $49.9 \pm 20.2(225)$ \\
\hline PASS, n/N (\%) & $36 / 223(16.1)$ & $27 / 225(12.0)$ \\
\hline \multicolumn{3}{|l|}{ SF-36 scores } \\
\hline Physical, mean \pm SD (n) & $38.6 \pm 7.5(216)$ & $37.4 \pm 7.7(216)$ \\
\hline Psychological, mean $\pm S D(n)$ & $46.6 \pm 10.0(216)$ & $46.2 \pm 11.5(216)$ \\
\hline \multicolumn{3}{|l|}{ Medication (at the time of inclusion) } \\
\hline At least one medication, $\mathrm{n} / \mathrm{N}(\%)$ & $110 / 223(49.3)$ & $117 / 228(51.3)$ \\
\hline NSAID, n/N (\%) & $33 / 223(14.8)$ & $37 / 228(16.2)$ \\
\hline SYSADOA, n/N (\%) & $58 / 223(26.0)$ & $52 / 228(22.8)$ \\
\hline Analgesic, n/N (\%) & $49 / 223(22.0)$ & $62 / 228(27.2)$ \\
\hline Hyaluronic acid, $\mathrm{n} / \mathrm{N}(\%)$ & $1 / 223(0.4)$ & $1 / 228(0.4)$ \\
\hline
\end{tabular}

PASS is the value beyond which patients can consider themselves well. It is composed of the VAS pain (cutoff $\leq 32 \mathrm{~mm}$ ), the WOMAC function scores (cutoff $\leq 31 \mathrm{~mm}$ ) and the patient's global self assessment of disease.

NSAID, non-steroidal anti-inflammatory drug: PASS, patient acceptable symptom state; SF36, Short Form 36; SYSADOA, symptomatic slow acting drug in osteoarthritis; VAS, visual analogue scale; WOMAC, Western Ontario and McMaster Universities osteoarthritis index.

$\alpha$ risk was $5 \%$, and the $\beta$ risk was $20 \%$. The number of patients was thus 58 per group per centre, or 67 allowing for $15 \%$ loss to follow-up.

The randomisation technique of Zelen was used. ${ }^{13} 14$ Eligible patients were randomly assigned to spa therapy or to the control group using a centralised computer programme. Randomisation was stratified by centre and in blocks of eight with random order. Concealment was assumed by a protected computer file.

The Zelen randomisation method ${ }^{14}$ implied that patients were not informed of the existence of two groups. If the patient refused to participate as randomised, they were offered the other treatment, but remained in their assigned group for intention to treat analysis.

In order to conceal the existence of the other group, ${ }^{14}$ randomisation was performed before written informed consent was obtained. Patients were told only about the group to which 
they had been assigned and were given one of the two possible patient information documents with the consent form. In addition, delocalisation of the consultation away from the spa setting was done with the intention of keeping patients ignorant of the other group.

\section{Statistics}

Analysis was performed by intention to treat. Categorical variables were expressed as frequency and percentage, continuous variables as mean and $\mathrm{SD}$. The main endpoint was tested using an uncorrected $\chi^{2}$ test. Risk ratios with $95 \% \mathrm{CI}$, odds ratios and $95 \%$ CI, number needed to treat and effect size with $95 \% \mathrm{CI}$ were calculated.

Secondary qualitative endpoints were analysed using the same principles. For continuous variables, an analysis of variance was performed for repeated data, assuming sphericity, (comparison M0-M6 and M0--M3-M6-M9) with a treatment factor and an interaction analysis (repetitions $\times$ treatment). Between-group comparisons at 6 months used Student's t test. For WOMAC and VAS pain, the effect size was equal to the mean change in score from baseline to 6 months, divided by the SD of the baseline score.

For subgroup analyses a Mantel-Haenszel test of homogeneity was used. For the WOMAC and SF36 scores, missing data for each subscale were replaced by the mean of all the patients who had replied to at least half the questions in that subscale, according to the recommendations for SF36. ${ }^{10}$

Two-sided $p$ values less than 0.05 were considered statistically significant. Analyses were performed using STATA software (version 10.0).

The trial protocol was passed favourably by the regional ethics committee (Lyon A) in April 2006 and registered on http:// www.clinicaltrials.gov with number NCT00348777. Study coordination, monitoring visits to each centre, data management, data entry from patient questionnaires and data analysis were performed by the Grenoble Clinical Research Centre.

\section{RESULTS}

The overall flow of patients included between June 2006 and April 2007 is shown in figure 1. In total, 7.2\% (16/223) patients

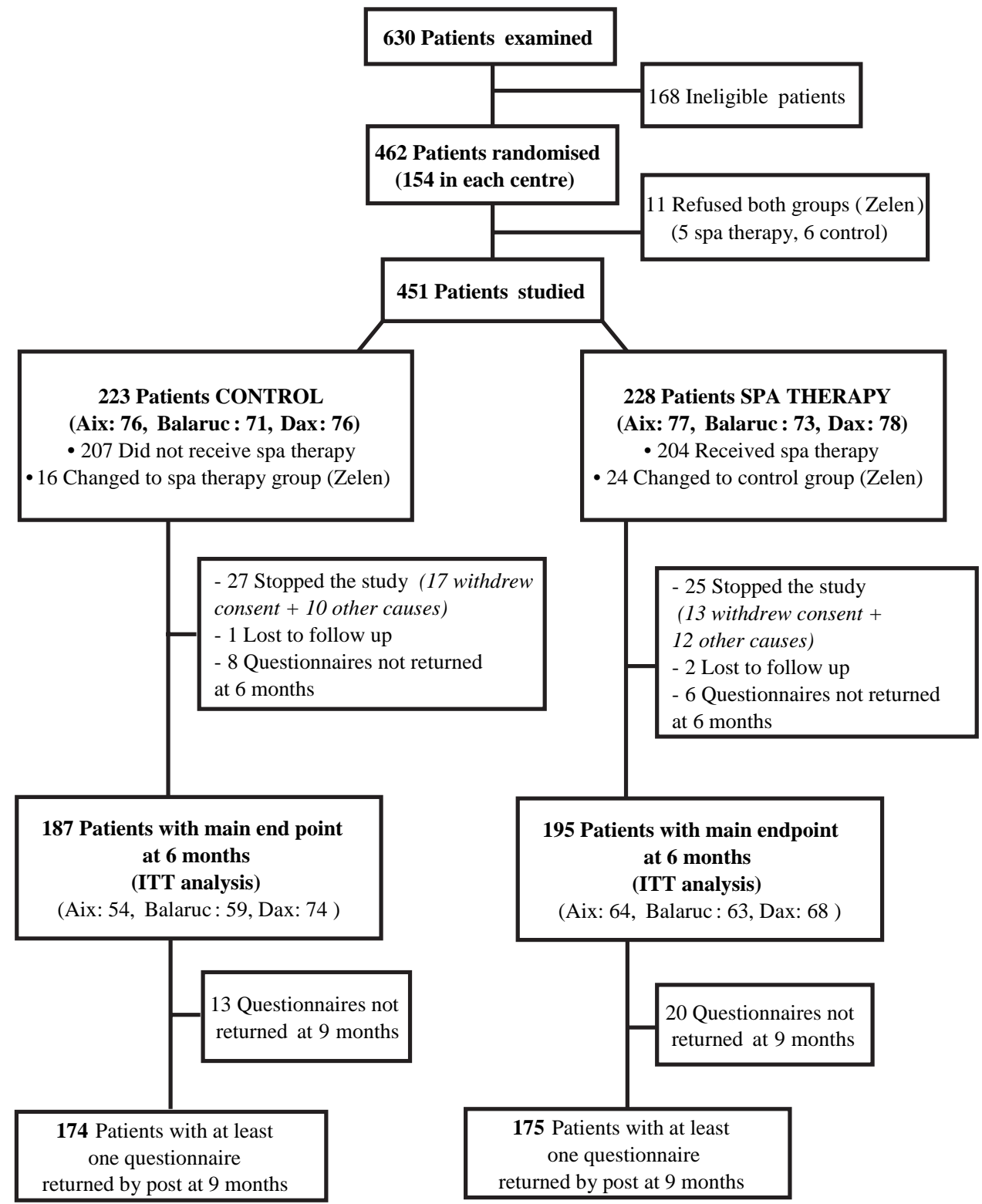

Figure 1 Study flow chart. ITT, intention to treat. 
Table 2 Number (\%) of patients achieving MCII at 3, 6 and 9 months

\begin{tabular}{llll}
\hline Visit & Control & Spa therapy & p Value \\
\hline 3 Months & $70 / 179(39.1 \%)$ & $107 / 183(58.5 \%)$ & \\
6 Months & $68 / 187(36.4 \%)$ & $99 / 195(50.8 \%)$ & 0.005 \\
Details & & & \\
$\quad$ Improvement of VAS pain* & $36 / 186(19.4 \%)$ & $63 / 193(32.6 \%)$ & \\
$\quad$ Improvement of WOMAC functiont & $49 / 172(28.5 \%)$ & $75 / 179(41.9 \%)$ & \\
9 Months & $62 / 173(35.8 \%)$ & $93 / 173(53.8 \%)$ & \\
\end{tabular}

MCll is defined as $\geq 19.9 \mathrm{~mm}$ on the VAS pain scale and/or $\geq 9.1$ points on the WOMAC function subscale normalised to a $0-100$ score and no knee surgery. *Three missing values for VAS pain.

†31 Missing values for WOMAC function.

MCII, minimal clinically important improvement; VAS, visual analogue scale; WOMAC, Western Ontario and McMaster Universities osteoarthritis index.

Table 3 Change in VAS pain and WOMAC scores (completed by the patient)

\begin{tabular}{|c|c|c|c|c|c|}
\hline & \multicolumn{5}{|c|}{ Difference: 6 months-inclusion } \\
\hline & \multicolumn{2}{|l|}{ Control } & \multicolumn{2}{|l|}{ Spa therapy } & \multirow[b]{2}{*}{ p Value } \\
\hline & Mean $\pm S D^{*}$ & $\begin{array}{l}\text { Effect size } \\
(95 \% \mathrm{CI})\end{array}$ & Mean \pm SD† & $\begin{array}{l}\text { Effect size } \\
(95 \% \text { CI) }\end{array}$ & \\
\hline VAS pain & $\begin{array}{l}-4.0 \pm 22.8 \\
(n=186)\end{array}$ & $\begin{array}{l}0.21 \\
(0.01 \text { to } 0.42)\end{array}$ & $\begin{array}{l}-11.4 \pm 24.9 \\
(n=193)\end{array}$ & $\begin{array}{l}0.55 \\
(0.35 \text { to } 0.75)\end{array}$ & 0.003 \\
\hline $\begin{array}{l}\text { WOMAC } \\
\text { function }\end{array}$ & $\begin{array}{l}-3.0 \pm 15.4 \\
(n=172)\end{array}$ & $\begin{array}{l}0.17 \\
(-0.04 \text { to } 0.38)\end{array}$ & $\begin{array}{l}-8.5 \pm 14.7 \\
(n=179)\end{array}$ & $\begin{array}{l}0.43 \\
(0.22 \text { to } 0.64)\end{array}$ & $<0.001$ \\
\hline
\end{tabular}

*Three missing values for VAS pain.

†31 missing values for WOMAC.

VAS, visual analogue scale; WOMAC, Western Ontario and McMaster Universities osteoarthritis index.

changed from the control to the spa group, and 10.5\% (24/228) changed from the spa group to the control group. Thirteen patients in the spa group withdrew consent to participate before starting the sessions. Of 12 who dropped out, mainly for health or family reasons, only two had started spa sessions. In the control group, 17 patients withdrew consent and another 10 failed to attend study visits.

\section{Main endpoint}

The difference in MCII at 6 months is statistically significant $\left(x^{2}=8.05 ; \mathrm{df}=1 ; \mathrm{p}=0.005\right)$ in favour of spa therapy (table 2$)$. Three patients in the spa group and one in the control group achieved MCII but underwent knee prosthesis surgery during the study. They were considered to be treatment failures. The risk ratio of MCII is 1.4 (95\% CI 1.1 to 1.8) for the spa group versus control, the odds ratio 1.8 (95\% CI 1.2 to 2.8 ), and the number needed to treat is 6.9 patients.

\section{Secondary endpoints}

The secondary endpoint results are internally coherent with the effect size found for the components of the main endpoint (tables 3 and 4 and further details online in supplementary file 2, available online only).

\section{Planned subgroup analyses, sensitivity analysis and unplanned post-hoc analyses}

See online supplementary file 2 (available online only).

\section{Adverse events}

One patient in the spa group was hospitalised for urinary lithiasis, no other unexpected serious adverse event was reported. For adverse events see supplementary file 2 , available online only.

\section{DISCUSSION}

This study demonstrates that an intensive course of spa therapy with a home exercise programme and usual treatment provides medium-term benefit over a home exercise programme and usual treatment alone in the management of knee osteoarthritis

To our knowledge this is the first multicentre RCT of spa therapy for knee osteoarthritis. The study size attains the number of patients (498) combined from seven smaller heterogeneous studies in a recent systematic Cochrane review. ${ }^{1}$

We used the Zelen randomisation method to blind patients in one group to the existence of the other group and to limit the level of dropout. It allowed patients assigned to spa therapy who did not want the constraint of having to attend a spa for 3 weeks, to change to the control group. The possibility to change could potentially reduce the difference between the two treatments as the intention to treat analysis was performed according to randomisation status.

We did not attempt to assess the efficacy of spa therapy alone, but only in combination with a home exercise programme and usual medical treatment. Both groups thus adhered to the current recommendations for the treatment of knee osteoarthritis. ${ }^{3}$ 1516 We minimised a 'placebo' effect in the control group, who were followed up in the same way as the spa group. ${ }^{17}$ The use of a qualitative endpoint measure, the MCII, may also reduce the influence of a potential 'placebo effect'. 18

Our main endpoint, MCII, is clinically relevant to the patient, comprising change in the WOMAC function subscale and VAS pain measurement, both recognised validated endpoint measures.

According to the recommendations for non-pharmacological trials, ${ }^{19}$ we used Zelen randomisation, employed examining physicians independent of the spa setting and patient self-assessed primary endpoint measures.

The amplitude of our result is in line with our hypothesis when we calculated the sample size needed for this study. Our effect sizes are similar to those for other treatments of knee osteoarthritis including hyaluronic acid, paracetamol and NSAID. ${ }^{20-22}$

Although only three patients were completely lost to follow-up, 52 patients withdrew consent or dropped out early on and 14 patients failed to return the questionnaires at 6 months, leading to a reduced number of patients or data in the main endpoint analysis at 6 months.

Our three centres receive approximately $30 \%$ of patients attending a spa in France. The standardised therapy delivered to osteoarthritis patients is similar to treatments delivered in other European spa settings.

The effect of spa therapy might be explained by that of a holiday. This was not the case here, patients had to drive daily to the spa, unlike those in a recent study by Karagülle et al. ${ }^{23}$ In France $26 \%$ of patients attend spa therapy on an outpatient basis, the rest staying on site for the duration of the course.

In general, our results confirm those already observed in other studies of a smaller size undertaken in a spa setting with natural hot mineral waters. Comparisons between the various studies are difficult as the baseline profiles of the patients are heterogeneous, the interventions differ in type, intensity and in length, ${ }^{24-33}$ the methods used for the assessment of efficacy vary and patients have been assessed at different time points after therapy.

At inclusion the severity of osteoarthritis in our patients was comparable to patients in the study advocating the use of MCII as an endpoint measure. ${ }^{11}$

This study attempts to assess the medium-term effect of spa therapy rather than simply short-term relief. Significant relief from pain at shorter time points after therapy compared with 
Table 4 Opinions of the patient and the physician at 6 months

\begin{tabular}{llll}
\hline & Control & Spa therapy & p Value \\
\hline Opinion of the patient & & & \\
Worse & $23 / 175(13.1 \%)$ & $12 / 180(6.7 \%)$ & $<0.001 ;$ \\
Neither worse nor better & $100 / 175(57.1 \%)$ & $70 / 180(38.9 \%)$ & $\chi^{2}=22.8 ; \mathrm{df}=2$ \\
Better & $52 / 175(29.7 \%)$ & $98 / 180(54.4 \%)$ & \\
Opinion of the examining physician & & \\
Worse & $12 / 175(6.9 \%)$ & $8 / 180(4.4 \%)$ & $<0.001 ;$ \\
$\quad$ Neither worse nor better & $109 / 175(62.3 \%)$ & $74 / 180(41.1 \%)$ & $\chi^{2}=20.2 ; \mathrm{df}=2$ \\
Better & $54 / 175(30.9 \%)$ & $98 / 180(54.4 \%)$ & \\
\hline
\end{tabular}

control is reported in other studies. ${ }^{27} 283234$ Our encouraging results obtained at 6 months appear to persist at least until 9 months.

What is the place of thermal spa therapy in the management of knee osteoarthritis? A recent high quality RCT demonstrated that arthroscopy of the knee has no lasting effect on knee osteoarthritis. ${ }^{35}$ Likewise, acupuncture plus a course of advice and a home exercise programme showed no significant effect of real acupuncture at 6 months follow-up. ${ }^{36}$ Spa therapy appears to provide some benefit.

A physical exercise regimen has been shown to be effective for knee osteoarthritis. Nevertheless, it is well known that unsupervised home exercise programmes often have limited efficacy over time as a result of progressive lack of compliance, even in the context of therapeutic trials with consecutive visits and that are motivating for the patients. ${ }^{47} \mathrm{~A}$ course of spa therapy may thus enhance patient compliance to the home exercise programme.

In conclusion, this RCT argues in favour of a clinical effect of spa therapy, as practised in France, for patients with knee osteoarthritis who continue their usual medical treatment and are encouraged to do regular exercises at home. In addition spa therapy is well tolerated.

Acknowledgements The authors thank Sandrine Massicot for data monitoring and Wainer Tabone, Eric Vignon and Claudine Rouffet for their suggestions. The authors also thank the examining physicians: Drs Y Attal, JC Perronnard and L Mendiharrat; the spa physicians and the secretaries in each centre, and the osteoarthritis group of the French Society for Rheumatology for their support (in particular Dr Michel Lequesne and Prs Serge Poiraudeau, Jean-François Maillefert and Xavier Chevalier).

Funding This study was funded by the French Society for Spa Research (Association Française pour la Recherche Thermale, AFRETH) a non-profit organisation, the RhoneAlpes Regional Council and the County Council of Savoie.

\section{Competing interests None.}

Ethics approval This study was conducted with the approval of the Ethics Committee Lyon A, Lyon, France.

Contributors RF (rheumatologist) initiated the project, wrote the protocol, organised data collection and wrote the article; HD (rheumatologist) organised data collection and contributed to writing the article; J-MT (rheumatologist) organised data collection and contributed to writing the article; AF (rheumatologist) contributed to the writing of the protocol and the article; AMF (medical writer and translator) translated, reviewed data interpretation, critically revised and edited the article; CG (statistician) performed the statistical analyses and critically read the article; CR (study coordinator) managed the study and critically read the article; C-FR (professor of physical medicine and rehabilitation) contributed to writing the article; J-LB (professor of medical statistics and coordinator of the Clinical Research Centre) contributed to writing the protocol, supervised the statistical analysis and contributed to writing the article.

Provenance and peer review Not commissioned; externally peer reviewed.

\section{REFERENCES}

1. Verhagen A, Bierma-Zeinstra S, Lambeck J, et al. Balneotherapy for osteoarthritis. A Cochrane review. J Rheumatol 2008;35:1118-23.

2. Jordan KM, Arden NK, Doherty M, et al. EULAR Recommendations 2003: an evidence based approach to the management of knee osteoarthritis: report of a
Task Force of the Standing Committee for International Clinical Studies Including Therapeutic Trials (ESCISIT). Ann Rheum Dis 2003;62:1145-55

3. Zhang W, Moskowitz RW, Nuki G, et al. OARSI recommendations for the management of hip and knee osteoarthritis. Part II: OARSI evidence-based, expert consensus guidelines. Osteoarthr Cartil 2008;16:137-62.

4. Davey R, Edwards SM, Cochrane T. Recruitment strategies for a clinical trial of community-based water therapy for osteoarthritis. Br J Gen Pract 2003:53:315-17.

5. Altman R, Asch E, Bloch D, et al. Development of criteria for the classification and reporting of osteoarthritis. Classification of osteoarthritis of the knee. Diagnostic and Therapeutic Criteria Committee of the American Rheumatism Association. Arthritis Rheum 1986;29:1039-49.

6. Huskisson EC. Measurement of pain. Lancet 1974;4:1127-31.

7. O'Reilly SC, Muir KR, Doherty M. Effectiveness of home exercise on pain and disability from osteoarthritis of the knee: a randomised controlled trial. Ann Rheum Dis 1999;58:15-19

8. Ravaud P, Giraudeau B, Logeart I, et al. Management of osteoarthritis (OA) with an unsupervised home based exercise programme and/or patient administered assessment tools. A cluster randomised controlled trial with a $2 \times 2$ factorial design. Ann Rheum Dis 2004;63:703-8.

9. Bellamy N, Buchanan WW, Goldsmith $\mathrm{CH}$, et al. Validation study of WOMAC: a health status instrument for measuring clinically important patient relevant outcomes to antirheumatic drug therapy in patients with osteoarthritis of the hip or knee. $J$ Rheumatol 1988;15:1833-40.

10. Devernay M, Ecosse E, Coste J, et al. Determinants of medical care for young women with Turner syndrome. J Clin Endocrinol Metab 2009;94:3408-13.

11. Tubach $\mathbf{F}$, Ravaud $P$, Baron $G$, et al. Evaluation of clinically relevant changes in patient reported outcomes in knee and hip osteoarthritis: the minimal clinically important improvement. Ann Rheum Dis 2005;64:29-33.

12. Tubach $\mathbf{F}$, Ravaud $P$, Baron $G$, et al. Evaluation of clinically relevant states in patient reported outcomes in knee and hip osteoarthritis: the patient acceptable symptom state. Ann Rheum Dis 2005;64:34-7.

13. Zelen M. A new design for randomized clinical trials. N Eng/ J Med 1979;31:1242-5

14. Zelen M. Randomized consent designs for clinical trials: an update. Stat Med 1990;9:645-56.

15. Fransen $\mathbf{M}, \mathbf{M c}$ Connell S, Bell M. Exercise for osteoarthritis of the hip or knee. Cochrane Database Syst Rev 2003;CD 004286

16. van Baar ME, Assendelft WJ, Dekker J, et al. Effectiveness of exercise therapy in patients with osteoarthritis of the hip or knee: a systematic review of randomized clinical trials. Arthritis Rheum 1999;42:1361-9.

17. Zhang $\mathbf{W}$, Robertson J, Jones AC, et al. The placebo effect and its determinants in osteoarthritis: meta-analysis of randomised controlled trials. Ann Rheum Dis 2008;67:1716-23.

18. Hróbjartsson A, Gøtzsche PC. Is the placebo powerless? An analysis of clinical trials comparing placebo with no treatment. N Engl J Med 2001;344:1594-602.

19. Boutron I, Tubach F, Giraudeau B, et al. Methodological differences in clinical trials evaluating nonpharmacological and pharmacological treatments of hip and knee osteoarthritis. JAMA 2003;290:1062-70.

20. Arrich J, Piribauer F, Mad P, et al. Intra-articular hyaluronic acid for the treatment of osteoarthritis of the knee: systematic review and meta-analysis. Can Med Assoc J 2005; 172:1039-43.

21. Neame R, Zhang W, Doherty M. A historic issue of the annals: three papers examine paracetamol in osteoarthritis. Ann Rheum Dis 2004;63:897-900.

22. Bjordal JM, Ljunggren $A E$, Klovning $A$, et al. Non-steroidal anti-inflammatory drugs, including cyclo-oxygenase-2 inhibitors, in osteoarthritic knee pain: meta-analysis of randomised placebo controlled trials. BMJ 2004;329:1317.

23. Karagülle M, Karagülle MZ, Karagülle 0, et al. A 10-day course of spa therapy is beneficial for people with severe knee osteoarthritis. A 24-week randomised, controlled pilot study. Clin Rheumatol 2007;26:2063-71

24. Kovács I, Bender T. The therapeutic effects of Cserkeszölö thermal water in osteoarthritis of the knee: a double blind, controlled, follow-up study. Rheumatol Int 2002;21:218-21.

25. Sukenik S, Flusser D, Codish S, et al. Balneotherapy at the Dead Sea area for knee osteoarthritis. Isr Med Assoc J 1999;1:83-5.

26. Green J, McKenna F, Redfern EJ, et al. Home exercises are as effective as outpatient hydrotherapy for osteoarthritis of the hip. Br J Rheumatol 1993;32:812-15.

27. Tishler M, Rosenberg 0, Levy 0, et al. The effect of balneotherapy on osteoarthritis. Is an intermittent regimen effective? Eur J Intern Med 2004;15:93-6.

28. Obadasi E, Karagülle MZ, Karagülle Z, et al. Comparison of two spa therapy regimens in patients with knee osteoarthritis, an exploratory study. Phys Med Rehab Kuror 2002;12:337-41.

29. Evcik D, Kavuncu V, Yeter A, et al. The efficacy of balneotherapy and mud-pack therapy in patients with knee osteoarthritis. Joint Bone Spine 2007;74:60-5.

30. Wigler I, Elkayam 0, Paran D, et al. Spa therapy for gonarthrosis: a prospective study. Rheumatol Int 1995;15:65-8.

31. Gaál J, Varga J, Szekanecz Z, et al. Balneotherapy in elderly patients: effect on pain from degenerative knee and spine conditions and on quality of life. Isr Med Assoc J 2008:10:365-9 
32. Nguyen M, Revel M, Dougados M. Prolonged effects of 3 week therapy in a spa resort on lumbar spine, knee and hip osteoarthritis: follow-up after 6 months. A randomized controlled trial. Br J Rheumatol 1997;36:77-81.

33. Forestier R. Magnitude and duration of the effects of two spa therapy courses on knee and hip osteoarthritis: an open prospective study in 51 consecutive patients. Joint Bone Spine 2000;67:296-304.

34. Odabasi E, Turan M, Erdem $\mathrm{H}$, et al. Does mud pack treatment have any chemical effect? A randomized controlled clinical study. J Altern Complement Med 2008;14:559-65.
35. Kirkley A, Birmingham TB, Litchfield RB, et al. A randomized trial of arthroscopic surgery for osteoarthritis of the knee. N Engl J Med 2008;359:1097-107.

36. Foster NE, Thomas E, Barlas P, et al. Acupuncture as an adjunct to exercise based physiotherapy for osteoarthritis of the knee: randomised controlled trial. BMJ 2007:335:436.

37. Campbell R, Evans M, Tucker M, et al. Why don't patients do their exercises? Understanding non-compliance with physiotherapy in patients with osteoarthritis of the knee. J Epidemiol Community Health 2001;55:132-8. 